\title{
A NEW METHOD FOR AUTOMATIC DETECTION OF THE ELECTROCARDIOGRAM CHARACTERISTIC POINTS
}

\section{Alejandro Castillo}

\begin{abstract}
In this article we propose a new method based on wavelet transform and on neural networks to detect the maximum, onset and offset of electrocardiogram (ECG) characteristic waves. First, the wavelet transform modulus maxima is used to extract main information of the ECG. We use Neural Networks to discern important modulus maxima and with them, the maximum point of a wave is detected. Then, a forward and backward search is made to detect the onset and offset of ECG waves. The detection degree of the maximum of the waves was proved using the QT Database and compared with another method. As a result, the proposed method can work with several morphologies of highly noisy ECGs.
\end{abstract}

Keywords: Electrocardiogram, ECG, Neural Networks, Wavelet Transform. 